\title{
Novel Role of Ras-GTPase Activating Protein SH3 Domain-Binding Protein G3BP in Adhesion and Migration of 32D Myeloid Progenitor Cells
}

\author{
Kerstin Schwarz ${ }^{1}$, Frank Aschenbrenner ${ }^{1}$, Brigitte Rüster ${ }^{2}$, Manuela Kampfmann ${ }^{1}$, Martina Komor ${ }^{1}$, \\ Wolf-Karsten Hofmann ${ }^{3}$, Martin Ruthardt ${ }^{1}$, Reinhard Henschler ${ }^{2}$ and Gesine Bug ${ }^{1, *}$ \\ ${ }^{I}$ Department of Medicine II, Hematology and Oncology, Johann Wolfgang Goethe University Frankfurt, Germany \\ ${ }^{2}$ Institute of Transfusion Medicine and Immune Hematology, German Red Cross Blood Donor Service Baden- \\ Württemberg - Hessen, Clinics of the Goethe University, Frankfurt, Germany \\ ${ }^{3}$ Department of Hematology and Oncology, Medical Faculty Mannheim of the University Heidelberg-Mannheim, Germany
}

\begin{abstract}
Rho GTPases are involved in homing and mobilization of hematopoietic stem and progenitor cells due to their impact on cytoskeleton remodeling. We have previously shown that inhibition of Rho, Rac and Cdc42 clearly impairs adhesion of normal and leukemic hematopoietic progenitor cells (HPC) to fibronectin and migration in a three-dimensional stromal cell model. Here, we identified the Ras GTPase-Activating Protein SH3 Domain-Binding Protein (G3BP) as a target gene of Rho GTPases and analysed its role in regulating HPC motility. Overexpression of G3BP significantly enhanced adhesion of murine 32D HPC to fibronectin and human umbilical vein endothelial cells, increased the proportion of adherent cells in a flow chamber assay and promoted cell migration in a transwell assay and a three-dimensional stromal cell model suggesting a strong impact on the cytoskeleton. Immunofluorescent staining of G3BP-overexpressing fibroblasts revealed a Rho-like phenotype characterized by formation of actin stress fibers in contrast to the Rac-like phenotype of control fibroblasts. This is the first report implicating a role for G3BP in Rho GTPase-mediated signalling towards adhesion and migration of HPC. Our results may be of clinical importance, since G3BP was found overexpressed in human cancers.
\end{abstract}

Keywords: G3BP, Rho GTPases, 32D progenitor cells, adhesion, migration, homing, toxin B, lethal toxin.

\section{INTRODUCTION}

Proteins of the Ras superfamily such as Ras, Rac, Rho, and $\mathrm{Cdc} 42$ have a determinant role in cell growth, differentiation, and malignant transformation and control cell adhesion and motility by remodelling of the cortical actin cytoskeleton [1]. GTPases are molecular switches that cycle between an active GTP-bound and an inactive GDPbound state. Activation is mediated by guanine nucleotide exchange factors (GEFs) that favor GTP binding while deactivation is regulated by GTPase-activating proteins (GAPs) that accelerate hydrolysis of GTP to GDP via a catalytic domain [2]. A major down-regulator of Ras, RasGAP, contains additional structural motifs including an SH3 domain, that is involved in cytoskeleton reorganization, cell adhesion and induction of gene expression in a Ras-dependent manner [3, 4]. The Ras-GTPase activating protein SH3 domain binding protein (G3BP) has been identified as one of only four proteins known to interact with RasGAP via its SH3 domain [5]. G3BP is composed of 466 amino acids and has a predicted molecular mass of $52 \mathrm{kDa}$. Three isoforms of the gene G3BP-1 and two alternatively spliced isoforms of mouse and human G3BP-2 (G3BP-2 $\alpha$ and G3BP-2 $\beta$ ) have been described. While the isoforms G3BP-2 $\alpha$ and $-2 \beta$ have been mapped to $4 \mathrm{q} 12-4 \mathrm{q} 24, \mathrm{G} 3 \mathrm{BP} 1$ is located on $5 \mathrm{q} 14.2-5 \mathrm{q} 33.3$ [6]. G3BP

\footnotetext{
*Address correspondence to this author at the Department of Medicine II, Hematology and Oncology, Johann Wolfgang Goethe University, TheodorStern-Kai 7, 60590 Frankfurt, Germany; Tel: +49-69-6301-7760; Fax: +49-69-6301-7790; E-mail: g.bug@em.uni-frankfurt.de
}

is overexpressed in several human cancer cell lines and various cancer tissues and stimulates S-phase entry in cultured cells [7]. In addition, G3BP expression has been positively correlated with the presence of lymph node metastasis and has been shown to promote invasion of cancer cells [8].

The bone marrow (BM) microenvironment constitutes a homing compartment for transplanted hematopoietic stem and progenitor cells. Homing is defined as the specific migration of circulating hematopoietic stem and progenitor cells through the vasculature to the $\mathrm{BM}$, which is a prerequisite for the engraftment in specialized BM niches that support and regulate maintenance, proliferation and differentiation of hematopoietic stem and progenitor cells $[9,10]$.

Previously, we investigated the contribution of different Rho GTPase members to migration of human CD34 ${ }^{+}$stem and progenitor cells: in a three-dimensional BM stromal cell environment, migration was significantly inhibited by cell permeable, Rho GTPase-blocking bacterial toxins: Toxin B derived from C. difficile inactivates Rho, Rac and Cdc42 [11], whereas lethal toxin of $C$. sordellii predominantly inactivates Rac and also to some degree $\mathrm{Cdc} 42$, but not Rho [12]. Here, we identify G3BP as one of several genes downregulated by toxin B and lethal toxin and characterize its impact on adhesion and migration of HPC.

\section{MATERIALS AND METHODOLOGY \\ Cell Culture and Chemicals}

RAT-1 cells were maintained in DMEM medium (Invitrogen, Karlsruhe, Germany) supplemented with $10 \%$ fetal calf se- 
rum (FCS; Hyclone, Greiner, Frickenhausen, Germany) with $1 \%$ glutamine $(200 \mathrm{mM})$ and $1 \%$ penicillin/streptomycin (Invitrogen). The murine myeloid progenitor cell line 32D (No. ACC411, DSMZ, Braunschweig, Germany) was cultured in RPMI 1640 (Gibco-Life Technologies, Paisley, UK) supplemented with $10 \%$ FCS and $10 \mu \mathrm{g} / \mathrm{ml}$ murine interleukin-3 (IL-3; Peprotech, London, UK). The human CD34 ${ }^{+}$cell line TF-1 (No. CRL-2003, American Type Culture Collection, Manassas, VA) was cultured in RPMI 1640 with $10 \%$ FCS in the presence of human IL-3 (R\&D Systems, Wiesbaden, Germany) and granulocyte macrophage-colony stimulating factor (GM-CSF; $10 \mathrm{ng} / \mathrm{ml}$ each; Essex Pharma, Munich, Germany). TF-1 cells were incubated with $100 \mathrm{ng} / \mathrm{ml}$ toxin B or lethal toxin in IMDM/10\% FCS for 6 hours at $37^{\circ} \mathrm{C}$ (toxins were kindly provided by H. Barth and K. Aktories, Institute of Pharmacology and Toxicology, University of Freiburg, Germany). The murine BM stroma cell line M2-10B4 (kindly provided by D. Hogge, Terry Fox Laboratory, Vancouver, Canada), modified to produce human IL-3 and GM-CSF, was maintained in RPMI 1640 with $10 \% \mathrm{FCS}, 0.06 \mathrm{mg} / \mathrm{ml}$ hygromycin B (Calbiochem, Bad Soden, Germany), $0.4 \mathrm{mg} / \mathrm{ml}$ geneticin, and $8 \mathrm{mM}$ HEPES (both from Gibco-Life Technologies). Human umbilical vein endothelial cells (HUVEC) were maintained in medium 199 (M199) with Earle's salts (Sigma Chemical, St. Louis, MO) supplemented with $10 \%$ heat-inactivated fetal bovine serum (FBS; Sigma-Aldrich, Steinheim, Germany), 1\% antibiotic-antimycotic solution (Sigma-Aldrich), $2 \mathrm{mM}$ L-glutamine, $120 \mu \mathrm{g} / \mathrm{ml}$ heparin (Sigma-Aldrich), and $100 \mu \mathrm{g} / \mathrm{ml}$ endothelial cell growth supplement (Collaborative Biomedical, Bedford, MA).

\section{Microarray Analysis}

Hybridization of total RNA to the HuGeneFL oligonucleotide microarray (Affymetrix Inc., Santa Clara, CA) was performed as described previously [13]. Briefly, at least 8 $\mu \mathrm{g}$ of total RNA were reverse transcribed by Superscript II reverse transcriptase (Invitrogene, Grand Island, NY) using T7- $(\mathrm{dT})_{24}$ primer containing a T7 RNA polymerase promoter site. After synthesis of the second cDNA strand, this product was used in an in vitro transcription reaction to generate biotinylated complementary cRNA. Fifteen micrograms of fragmented cRNA was hybridized to a $\mathrm{HuGeneFL}$ microarray for 16 hours at $45^{\circ} \mathrm{C}$ with constant rotation at $60 \mathrm{rpm}$ according to the Affymetrix protocol. The fluorescence intensity was scanned by the Affymetrix GeneChip Scanner and normalized by global scaling to the average fluorescence intensity for the entire microarray. Data analysis was performed with the GeneSpring software version 4.2 (Silicon Genetics, San Carlos, CA) and Microarray Analysis Suites 4.01 (MAS 4.01, Affymetrix, Inc.). Selection of differentially expressed genes required at least a 2-fold change in normalized expression values.

\section{Cloning of Full-Length G3BP}

A 1412 bp cDNA encoding full-length G3BP of human origin was obtained by PCR cloning using the One-step RT-PCR Kit (Invitrogen) and specific oligonucleotides G3BP_fwd (5'-AGCAATGGTGATGGAGAAGCC-3') and G3BP_rev (5'- CATGAAGATTACTGCCGAGGA-3'). The PCR product was cloned into the pCR2.1 vector with the TA-cloning kit (Invitrogen) according to the manufacturer's instructions. An expression vector for G3BP was produced using the Gateway recombination technology (Invitrogen). Briefly, the cDNA encoding full-length G3BP was first subcloned into pENTR1A using an EcoRI restriction site and then transferred into the GFP expressing destination vector pinco [14] by LR recombination following the manufacturer's instructions (Invitrogen). The resulting vectors are referred to as control (pinco empty vector) and pinco_G3BP (full length G3BP in pinco vector).

\section{Retroviral Infection}

Ecotropic Phoenix packaging cells were transiently transfected with the indicated retroviral vectors as described before [15]. For the infection target cells were plated onto retronectincoated (Takara-Shuzo, Shiga, Japan) non-tissue culture treated 24-well plates and exposed to the retroviral supernatant for 3 hours at $37^{\circ} \mathrm{C}$ in the presence of $4 \mu \mathrm{g} / \mathrm{mL}$ polybrene (SigmaAldrich). Cells were centrifuged at 2,200 rpm for 45 minutes. Infection was repeated four times and infection efficiency had to be at least $70 \%$ for each sample as assessed by detection of green fluorescent protein-positive cells by FACS.

\section{Small Hairpin RNA}

The small hairpin RNA (shRNA) sequences encoding inverted repeats of 21 nucleotides (nts) separated by a 10-nt spacer were designed using publicly available software tools (www.ambion.com/techlib/misc/silencer_siRNA_template.html). Three shRNAs against different regions of the G3BP sequence were tested. The inverted repeats corresponded to bp 173-194 (shRNA1), bp 450-471 (shRNA2) and bp 679-700 (shRNA3) of the murine G3BP cDNA and had at least 3 nt differences from any other murine gene. A shRNA derived from prokaryotic lacZ gene served as unspecific control. The oligos containing $\mathrm{HpaI}$ and $\mathrm{BbsI}$ restriction sites and hairpin DNA were annealed and ligated into the BbsI-HpaI-digested expression vector vPGKpuroU6FH [15]. The construct was controlled by sequencing and designated as si1-3. Retroviral infection of $32 \mathrm{D}$ cells was carried out as described above. Efficiency of the shRNA was confirmed by Western blotting.

\section{Western Blotting}

Western blotting was done according to widely used protocols with the following antibodies: anti-G3BP (BD Transduction Laboratories, San Diego, USA) and anti- $\alpha$-tubulin (Dianova, Hamburg, Germany). All antibodies were diluted in 5\% low fat dry milk. Blocking was performed in 5\% low fat dry milk, washing was carried out in TBS containing $0.1 \%$ Tween20 (TBS-T). Densitometry was performed using Quantity One Software from BioRad (Munich, Germany).

\section{Cell Adhesion Assay}

Fibronectin (FN, $5 \mu \mathrm{g} / \mathrm{cm}^{2}$; BD Biosciences, Heidelberg, Germany) diluted in PBS was adsorbed to wells of 6-well plates overnight at $4^{\circ} \mathrm{C}$. Non-specific bindings were blocked with PBS containing $2 \% \mathrm{BSA}$ for 1 hour at $37^{\circ} \mathrm{C}$. RAT-1 or 32D cells were washed once in PBS and plated at $1 \times 10^{6}$ cells in 3 $\mathrm{ml}$ DMEM with $10 \%$ FCS. Cells were allowed to adhere to the coated plates for 3 hour at $37^{\circ} \mathrm{C}$ in a humidified atmosphere of $5 \% \mathrm{CO}_{2}$. After incubation, non adherent cells were washed away with PBS by gentle agitation and adherent cells were counted.

\section{Flow Chamber Assay}

HUVEC of the second or third passage were grown to subconfluency on glass slides and activated by TNF- $\alpha(100 \mathrm{U} / \mathrm{ml})$ 
for 7 hours prior to the assay. The glass slides were assembled as the lower wall of the flow chamber (Circular Parallel Plate Flow Chamber kit; GlycoTech, Gaithersburg, MD, USA) and the flow chamber was mounted on the stage of an inverted phase-contrast microscope (Carl Zeiss, Jena, Germany). 32D cells $\left(10^{6}\right.$ cells $/ \mathrm{ml}$; mock-transfected or pinco_G3BP) were perfused through the chamber at the desired flow rate generated with an automated syringe pump (B. Braun Medical, Emmensbruegge, Switzerland). Initial application of the cells was performed with a shear stress of $0.1 \mathrm{dyn} / \mathrm{cm}^{2}$. After $10 \mathrm{~min}$, shear stress was increased to $2 \mathrm{dyn} / \mathrm{cm}^{2}$ for $20 \mathrm{~min}$. Subsequent to each perfusion period, adherent cells were documented by photographs of three independent fields using a gauged grid and counted [16].

\section{Transwell Assay}

Transwell units ( $8 \mu \mathrm{M}$ pore size; BD Falcon, Heidelberg, Germany) were coated with FN $\left(5 \mu \mathrm{g} / \mathrm{cm}^{2}\right)$ diluted in PBS over night at $4{ }^{\circ} \mathrm{C}$. RAT- 1 cells or $32 \mathrm{D}$ cells were washed once in PBS and seeded in the upper chamber at $1 \times 10^{4}$ cells in the adequate medium. Cells were allowed to spontaneously migrate through the pores of the membrane for 3 hours at $37^{\circ} \mathrm{C}$. After incubation, filters were washed with PBS by gentle agitation and migrated cells attached to the lower surface of the membrane were fixed with $3.7 \%$ formaldehyde and stained with coomassie staining solution (2\% Coomassie Brilliant Blue; $45 \%$ methanol; $10 \%$ acetic acid). Stained cells were documented by photographs of three independent fields and counted.

\section{Spheroid Assay}

M2-10B4 spheroids were grown in 1\% agarose-coated 96-well plates as previously described [17]. Briefly, $2.5 \times 10^{4}$ cells were inoculated per well in $200 \mu$ Iscove's modified Dulbecco's medium (IMDM; Biochrom, Berlin, Germany) supplemented with $10 \%$ FCS. After 4 days, $1 \times 10^{4} 32 \mathrm{D}$ cells were added for cocultivation. Spheroid co-cultures were harvested 24 hours later, washed with PBS, and dissociated with a $0.25 \%$ trypsin and $0.1 \%$ EDTA solution (1:3 in PBS; PAN Biotech, Aidenbach, Germany). Cell suspensions were filtered and incubated with FITCconjugated anti-human CD45 Ab (or anti-human IgG1Ab as control) to determine the percentage of hematopoietic cells in the spheroids. Analysis was performed on FACScan (BD Bioscience) using CellQuest and PC-Lysis software.

\section{Immunofluorescent Staining of Actin Cytoskeleton}

$2 \times 10^{4}$ RAT-1 cells were transduced with bicistronic retroviral vectors expressing the enhanced green fluorescent protein (GFP) and full-length G3BP (pinco_G3BP) or GFP alone (pinco_control) and were seeded on fibronectin-coated $(50 \mu \mathrm{g} / \mathrm{ml})$ coverslips and allowed to adhere for 3 hours at $37^{\circ} \mathrm{C}$. Non adherent cells were removed by gentle washing with PBS and adherent cells were fixed with $3.7 \%$ formaldehyde/PBS for $15 \mathrm{~min}$ at room temperature. After washing with PBS cells were permeabilized for 10 min with PBS containing $0.5 \%$ Triton-X. After blocking with AbDil solution (0.1\% Triton $\mathrm{X}$ in $\mathrm{PBS}, 2 \% \mathrm{BSA}, 0.1 \%$ sodiumacide) actin cytoskeleton was stained using phalloidin-TRITC $(0.5 \mu \mathrm{g} / \mathrm{ml}$ in AbDil; Sigma-Aldrich).

\section{Statistical Analysis}

Data were compared by a two-tailed Student $t$ test; $p$ values $<0.05$ were considered to be significant.

\section{RESULTS}

\section{G3BP is a Target Gene of Rho GTPases}

The cell permeable toxins from Clostridium spec. lethal toxin and toxin B have previously been shown to significantly impair migration, homing and engraftment of hematopoietic stem cells $[17,18]$. To further elucidate the molecular mechanisms of this inhibition, we treated human $\mathrm{CD}^{+} 4^{+} \mathrm{TF}-1$ cells with lethal toxin or toxin $\mathrm{B}$ and analyzed changes in gene expression using high-density microarrays. Within a list of 48 and 116 genes showing differential expression after treatment with toxin B or lethal toxin, respectively, we identified several genes regulated by both toxins implicated in cellular motility, cell-cell contact or GTPase signalling which are listed in Table 1. We focussed on RasGAP SH3 domain binding protein (G3BP) since the encoding gene is located on the long arm of chromosome 5, a chromosomal region frequently deleted in myelodysplastic syndromes and acute myeloid leukemia. The observed 2.5-fold downregulation of G3BP mRNA in toxintreated TF-1 cell compared to control cells was validated on the protein level by Western blot analysis using a monoclonal anti-G3BP antibody Fig. (1).

Table 1. Genes Regulated by Both Toxin B and Lethal Toxin (Identified by Gene Expression Profiling of Toxin Treated TF-1 Cells)

\begin{tabular}{|c|c|c|c|c|}
\hline Gene & Genbank & $\begin{array}{c}\text { Toxin B } \\
\text { Fold Change }\end{array}$ & $\begin{array}{c}\text { Lethal Toxin } \\
\text { Fold Change }\end{array}$ & Description \\
\hline \hline G3BP & U32519 & $2.5(-)$ & $2.7(-)$ & $2.3(-)$ \\
\hline DCN & M14219 & $2.1(-)$ & $3.1(-)$ & Capping protein (actin filament) muscle Z-line, alpha 2 \\
\hline CAPZA2 & U03851 & $2.3(-)$ & $2.3(-)$ & Pecorin \\
\hline P311 & U30521 & $1.6(-)$ & $2.0(-)$ & CD164 antigen, sialomucin \\
\hline CD164 & D14043 & $1.2(-)$ & $3.1(-)$ & IQ motif containing GTPase activating protein 1 \\
\hline IQGAP1 & L33075 & $1.1(-)$ & $2.7(+)$ & Plectin 1, intermediate filament binding protein, 500kD \\
\hline PLEC1 & Z54367 & $3.6(+)$ & & Protein SH3-domain-binding protein \\
\hline
\end{tabular}




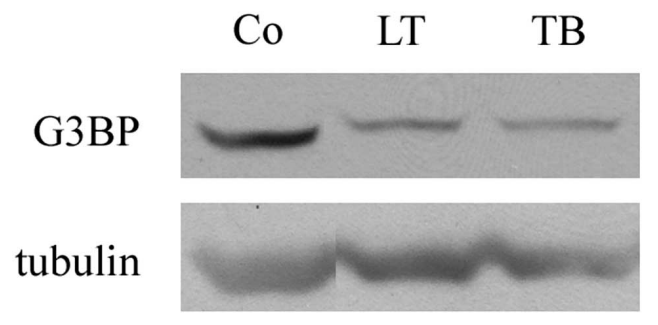

Fig. (1). Inhibition of small GTPases with Clostridium toxin B and lethal toxin inhibits expression of G3BP. TF-1 cells were treated for 6 hours with lethal toxin (LT) or toxin B (TB) to inhibit small GTPases of the Rho family. Western blot analysis of total cell lysates from treated cells or untreated controls (Co) was performed and anti-G3BP antibody (1:500) was used to determine G3BP expression level. Inhibition of GTPases by Clostridium spec. toxins markedly downregulated expression of G3BP protein. Tubulin was used as a loading control.

\section{G3BP Enhances Adhesion of 32D Progenitor Cells Under Shear Stress}

Having identfied G3BP as a target gene involved in Rho GTPase signalling in human hematopoietic progenitor cells (HPC) we evaluated its impact for the homing of HPC to the bone marrow. The BM endothelium is the first an-

A

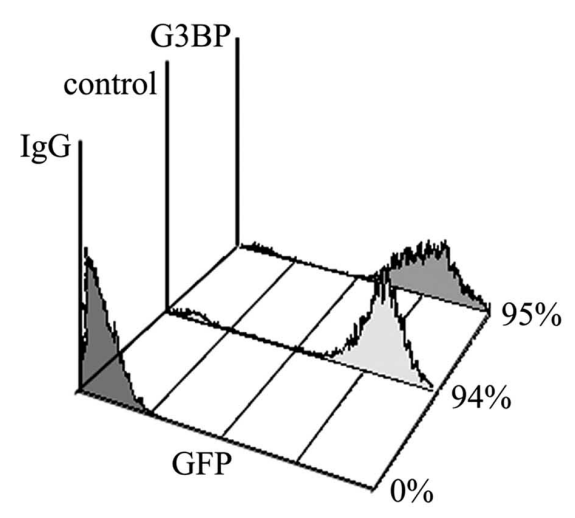

B

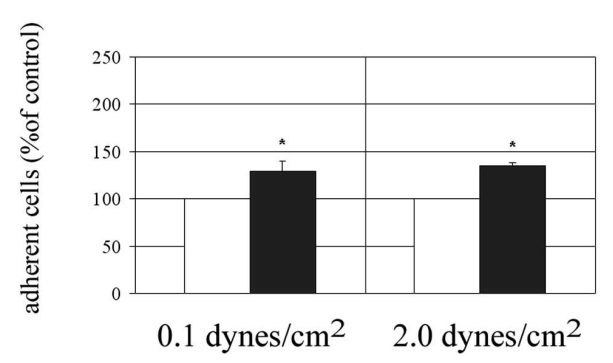

choring site for homing cells, supporting rolling and firm adhesion of circulating leukocytes by constitutive expression of adhesion molecules and stimulating cytokines [9]. We thus studied the interaction of murine 32D progenitor cells with HUVEC, a well-established vascular endothelial cell system [19]. 32D cells were infected with bicistronic retroviral vectors expressing the enhanced green fluorescent protein (GFP) and full-length G3BP (pinco_G3BP) or GFP alone (pinco_control) with an efficiency of $>90 \%$ as determined by FACS Fig. (2A) and perfused over a HUVEC monolayer in a flow chamber applying low and elevated shear stress. Cells adhering to the endothelial cell monolayer were documented and counted as described after $10 \mathrm{~min}$ at $0.1 \mathrm{dyn} / \mathrm{cm}^{2}$ and after another $20 \mathrm{~min}$ at $2 \mathrm{dyn} / \mathrm{cm}^{2}$. G3BP increased the number of rolling HPC by $23 \pm 16 \%\left(\mathrm{n}=4 ; p<0.05 ; 0.1 \mathrm{dyn} / \mathrm{cm}^{2}\right)$ and firmly adhering cells by $28 \pm 15 \%\left(\mathrm{n}=4 ; p<0.05 ; 2 \mathrm{dyn} / \mathrm{cm}^{2}\right)$ compared to mock-transfected controls Fig. (2B).

TNF-activated HUVEC support rolling and firm attachment of HPC under physiological shear-flow by displaying high levels of E-selectin, ICAM-1, and VCAM-1 [20]. As VCAM-1 is a vascular ligand for the integrin very late antigen (VLA)-4, we studied the effect of G3BP overexpression on HPC adhesion on fibronectin, one of the major extracellular matrix (ECM) component containing binding sites for VLA-4 (CD49d/CD29) and VLA-5 (CD49e/CD29). G3BP signifi-

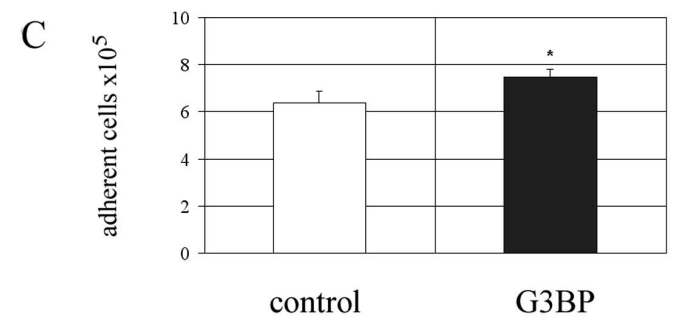

$\mathrm{D}$

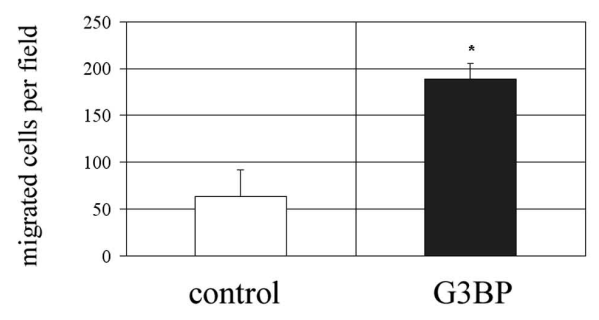

$\mathrm{E}$

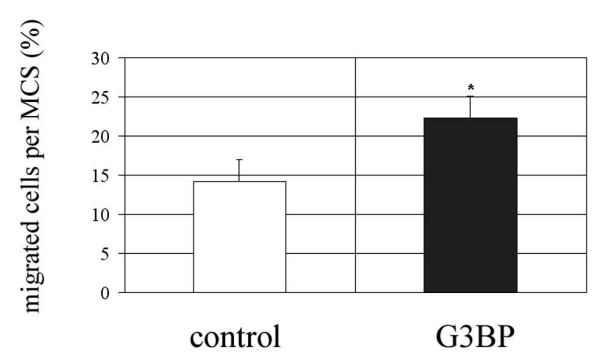

Fig. (2). Impact of G3BP on integrin-mediated adhesion and migration of HPC. Infection efficiency confirmed by FACS measurement of GFP-positive cells was $>90 \%$ in mock- and G3BP-transduced cells (A). Adhesion of 32D to a cellular endothelial surface (HUVEC monolayer) under low and elevated shear stress was tested in a flow chamber assay. G3BP significantly enhanced the number of firmly arrested cells compared to controls by $23 \pm 16 \%$ at $0.1 \mathrm{dyn} / \mathrm{cm}^{2}$ and $28 \pm 15 \%$ at $2.0 \mathrm{dyn} / \mathrm{cm}^{2}(\mathrm{n}=4)(\mathbf{B})$. G3BP was able to induce adhesion of $32 \mathrm{D}$ cells to fibronectin compared to controls $\left(7.5 \times 10^{5} \pm 0.5 \times 10^{5}\right.$ vs. $6.4 \times 10^{5} \pm 0.2 \times 10^{5}$ adherent cells, $\left.\mathrm{n}=4\right)(\mathbf{C})$. G3BP increased the number of migrated 32D cells from $63 \pm 29$ to $189 \pm 16$ cells/ field $(n=4)(D)$. Migration of 32D cells into multicellular spheroids $($ MCS $)$ was also increased by G3BP. The number of cells incorporated into the spheroids increased significantly from $14.1 \pm 2.8 \%$ to $22.3 \pm 2.8 \%$ ( $\mathrm{n}=4$ ) (E). $* P<0.05$. 
cantly enhanced adhesion of 32D cells to fibronectin compared to the corresponding controls Fig. (2C); $7.5 \times 10^{5} \pm$ $0.5 \times 10^{5}$ vs. $6.4 \times 10^{5} \pm 0.2 \times 10^{5}$ adherent cells, $\mathrm{n}=4 ; p<$ 0.05 . Enhanced interaction of HPC with ECM may improve transendothelial migration and trafficking within the BM microenvironment [9]. G3BP significantly induced spontaneous migration of $32 \mathrm{D}$ cells through the pores of a fibronectin-coated transwell filter resulting in $189 \pm 16 \mathrm{vs}$. $63 \pm 29$ migrated cells/field Fig. (2D), $\mathrm{n}=4 ; p<0.05$. Our data provide the first evidence that G3BP contributes to the regulation of adhesion and migration of HPC.

\section{G3BP Stimulates Migration of 32D Progenitor Cells into Stromal Cell Spheroids}

Multicellular spheroids (MCS) serve as a model for engraftment in the stem cell microenvironment. We have previously shown that migration of human $\mathrm{CD} 34^{+}$stem and progenitor cells in a three-dimensional BM stromal cell environment is significantly inhibited by cell permeable Rho GTPase-blocking bacterial toxins [17] Since G3BP proved to be a target gene downregulated upon toxin treatment we aimed to confirm the impact of G3BP on motility of murine 32D cells by comparing G3BP- to mock-infected controls. Migration was quantified by counting the number of cells incorporated in the spheroids. G3BP enhanced the number of incorporated HPC significantly from $14.1 \pm 2.8 \%$ to $22.3 \pm 2.8 \%(\mathrm{n}=4 ; p<0.05$; Fig. (2E). These results support the data obtained in the transwell assay and assures a role of G3BP in the regulation of migration of HPC.

A

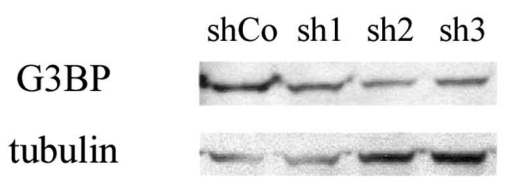

$\mathrm{C}$

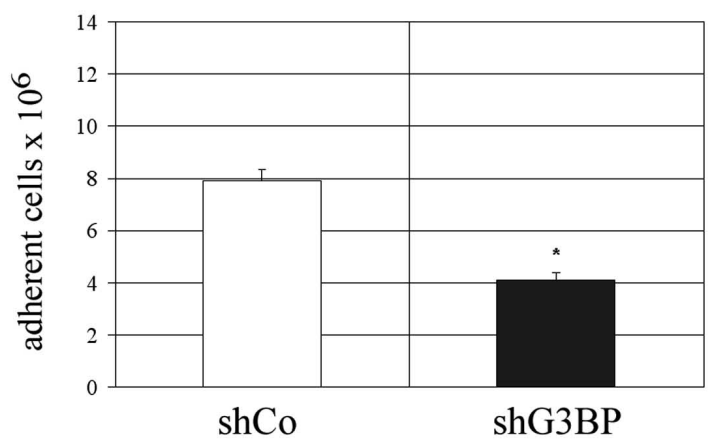

The impact of G3BP on adhesion and migration in 32D cells shown above was confirmed by shRNA experiments. All three shRNA vectors (sh1-3) applied were able to suppress protein expression in 32D cells as shown by western blotting Fig. (3A). Sh 2 resulted in a $75 \%$ reduction of G3BP protein compared to the control vector and was chosen for further experiments Fig. (3B). Suppression of G3BP by sh2 lead to a significantly decreased adhesion compared to controls (4.1 \pm $0.3 \times 10^{5}$ vs. $7.9 \pm 0.5 \times 10^{5}$ adherent cells; $p<0.05$; Fig. (3C). As expected, shRNA accordingly inhibited migration in the transwell assay in a significant way $230 \pm 70$ vs. $127 \pm 16$ migrated cells/field; $p<0.05$; Fig. (3D). These data confirm that G3BP plays an essential role in adhesion and migration of HPC.

\section{G3BP Induces Cytoskeletal Rearrangements in RAT-1 Cells}

The RasGAP SH3 domain is involved in cytoskeletal reorganization and cell adhesion [3,4]. We thus aimed to study the effect of G3BP on cytoskeletal rearrangements by comparing the morphology of RAT-1 cells infected with pinco_G3BP to mock-infected control cells Fig. (4A). Control cells spread on the fibronectin coated surface and displayed formation of lamellipodia at the edges Fig. (4B, upper panel). Actin fibers are visible but not organized in stress fibers. In contrast, overexpression of G3BP led to a dramatic induction of stress fibers Fig. (4B, lower panel) and increased the number of RAT-1 fibroblasts adhering to a fibronectin-coated surface from $5.2 \mathrm{x}$ $10^{5} \pm 0.1 \times 10^{5}$ to $7.5 \times 10^{5} \pm 0.2 \times 10^{5}$ when counted 3 hours after plating Fig. (4C, $\mathrm{n}=3, p<0.05)$. Our findings are consis-

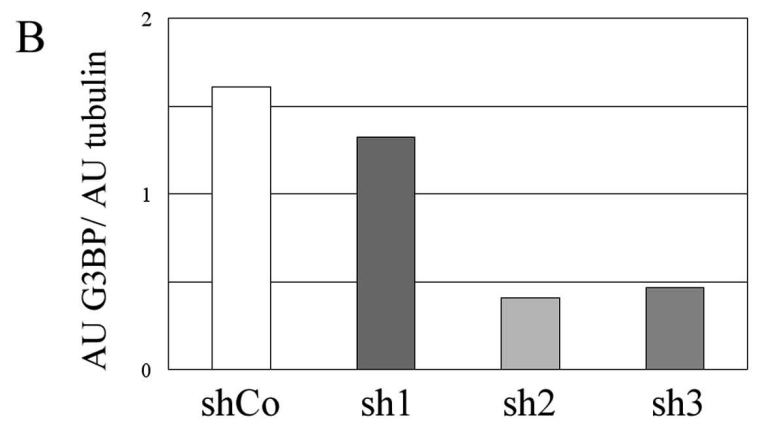

$\mathrm{D}$

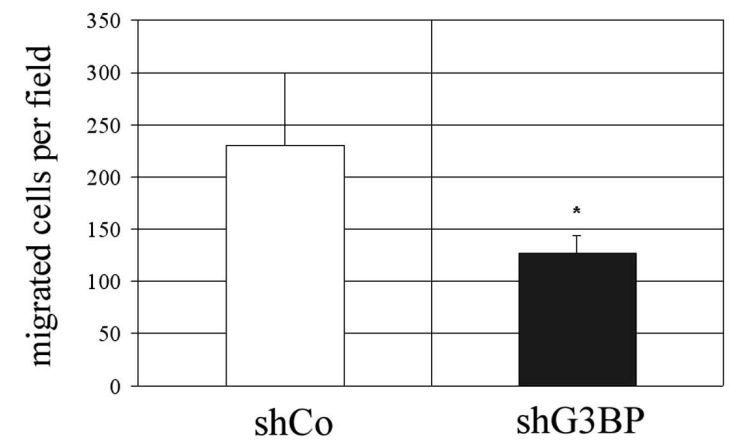

Fig. (3). RNAi of G3BP suppresses adhesion and migration in 32D cells. Three shRNA vectors (si1-3) applied were able to suppress G3BP expression in 32D cells as shown by Western blotting (A). The most effective one (si2) was chosen for further experiments verified by densitometry of the Western Blot shown above $(\mathbf{B})$. The suppression of G3BP by introduction of si2 lead to a significantly reduced adhesion compared to controls $\left(4.1 \pm 0.3 \times 10^{5}\right.$ vs. $7.9 \pm 0.5 \times 10^{5}$ adherent cells; $\left.\mathrm{n}=3\right)(\mathbf{C})$. As expected, RNAi significantly inhibited migration in the transwell assay ( $230 \pm 70$ vs. $127 \pm 16$ migrated cells/field; $n=3)(D) . * P<0.05$. 
A

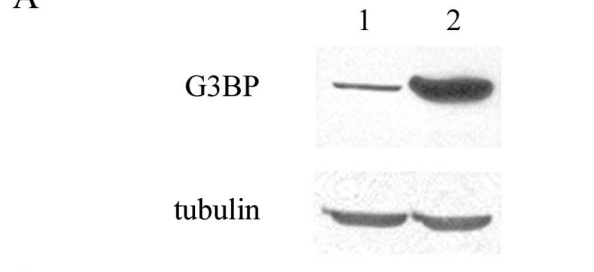

$\mathrm{C}$

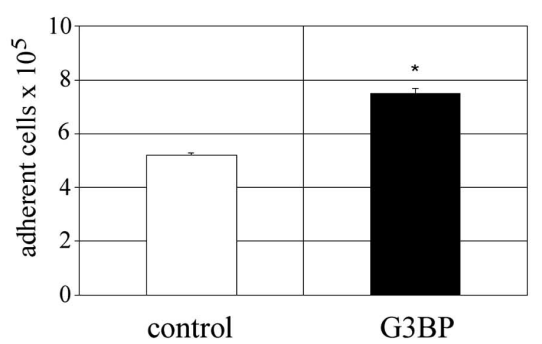

B
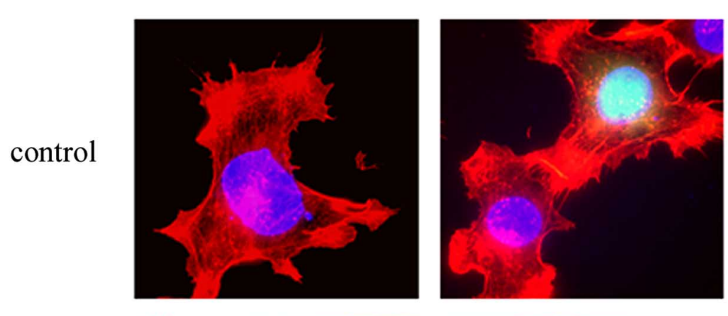

G3BP
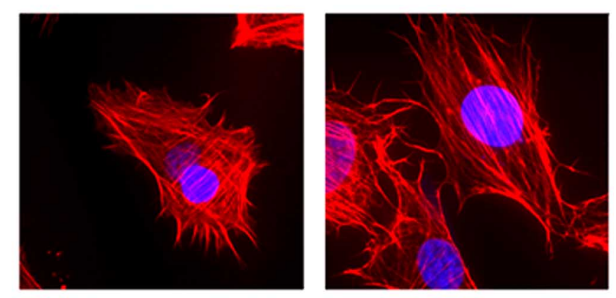

Fig. (4). Retroviral overexpression of G3BP in RAT-1 fibroblasts. Full length G3BP was cloned in the retroviral expression vector pinco_GFP. The viral construct successfully infected RAT-1 fibroblasts. Overexpression of G3BP was confirmed by western blotting (A). G3BP induces formation of actin stress fibers (lower panel) while mock-transfected controls (upper panel) display more lamellipodia-like protrusions $(\mathbf{B})$. Adhesion of G3BP overexpressing cells $(\boldsymbol{\square})$ was compared to mock-infected controls ( $\square$ ) as described. After 3 hours, a significantly higher number of adherent G3BP-overexpressing cells was counted compared to controls $\left(\mathbf{C} ; 7.5 \times 10^{5} \pm 0.2 \times 10^{5}\right.$ vs. $5.2 \times 10^{5}$ $\left.\pm 0.1 \times 10^{5}, \mathrm{n}=3\right) . * P<0.05$.

tent with the previously described Rho-like phenotype in resting fibroblasts upon overexpression of RasGAP [3].

\section{DISCUSSION}

Suppression of migration, short-term homing, hematopoietic cell regeneration and cell cycling by toxin $\mathrm{B}$ and lethal toxin has been reported $[17,18]$, demonstrating impairment of stem cell functions by both toxins, similar to that published for selective inhibition of Rac and $\mathrm{Cdc} 42$. Here, we provide evidence that the Ras GTPase activating protein SH3 domain-binding protein (G3BP) is significantly downregulated by toxin $\mathrm{B}$ and lethal toxin. Adhesion and migration of HSC are considered to be important components of efficient HSC homing, a prerequisite of therapeutic stem cell transplantation. In order to home to the BM, HPC have to roll along and firmly adhere to the blood vessel, cross the endothelium barrier and finally traffic within the BM microenvironment. [9] We utilized a variety of appropriate in vitro adhesion and migration assays to study the impact of G3BP on each sequential homing step $[17,20,21]$. Overexpression of G3BP in the murine HPC line 32D enhanced rolling and adhesion to endothelial cells under physiological shear flow, improved transmigration through a fibronectin-coated permeable membrane and enhanced migration into three-dimensional spheroids composed of BM stromal cells. We further demonstrate that knock-down of endogenous G3BP by shRNA caused impaired adhesion and migration of murine 32D HPC. Thus, our results indicate that at least a part of the toxin-induced effects on HPC are attributable to G3BP. Similar effects have been observed by inhibition of Rac or RhoA in primary HPC [22]. Furthermore, deletion of both Rac1 and Rac 2 causes massive defects in stem cell proliferation and survival in vitro and Rac1-deficient stem cells have reduced homing efficiency to the $\mathrm{BM}$ and fail to engraft $[23,24]$.

Integrin-dependent activation of Rho GTPases has been extensively studied in fibroblasts plated on fibronectin.
Engagement of integrins with ECM leads to activation of Cdc42 and subsequently Rac. Together, these GTPases mediate cell spreading and membrane ruffling, while Rho is activated independently to induce stress fibers [25,26]. In this setting, overexpression of G3BP in RAT-1 fibroblasts produced a Rho-like phenotype characterized enhanced formation of stress fibers and lead to extension of thin processes instead of lamellipodia consistent with inhibition of Rac. This is in agreement with data demonstrating that RasGAP per se is able to trigger stress fiber formation by stimulating Rho activity whereas blocking of the SH3 domain of RasGAP specifically abrogates Rho-dependent cytoskeletal reorganization [3]. Additional results obtained with the Drosophila homologue of G3BP suggest a role in both Ras and Rho signalling by serving as a link between these two GTPases [27].

\section{CONCLUSION}

In conclusion, we identify G3BP as one of several genes significantly downregulated by toxin $\mathrm{B}$ and lethal toxin and for the first time define its major contribution to adhesion and motility of 32 D HPC and cytoskeletal alterations in fibroblasts.

\section{CONFLICT OF INTEREST}

The authors declare no conflict of interest.

\section{ACKNOWLEDGEMENTS}

This work was supported by a grant of the Deutsche José Carreras Leukämie-Stiftung e.V. to GB (DJCLS R03/12). We gratefully thank Dr. Holger Barth and Prof. Dr. Klaus Aktories for the gift of the bacterial toxins.

\section{AUTHORS' CONTRIBUTIONS}

KS carried out the molecular biology studies and the immunfluorescent staining, participated in the functional assays and helped to draft the manuscript. FA MKampfmann participated in the molecular biology studies and the functional assays. BR carried out the functional assays. MKomor and 
WKH performed the microarray analysis. MR participated in the molecular biology studies and the immunfluorescent staining and helped to draft the manuscript. $\mathrm{RH}$ participated in the functional assays and helped to draft the manuscript. GB designed and coordinated the experiments and drafted the manuscript.

\section{REFERENCES}

[1] Etienne-Manneville S, Hall A. Rho GTPases in cell biology. Nature 2002; 420: 629-35.

[2] Boguski MS, McCormick F. Proteins regulating Ras and its relatives. Nature 1993; 366: 643-54.

[3] Leblanc V, Tocque B, Delumeau I. Ras-GAP controls rhomediated cytoskeletal reorganization through Its SH3 Domain. Mol Cell Biol 1998; 18: 5567-78.

[4] Medema RH, de Laat WL, Martin GA, McCormick F, Bos JL GTPase-activating protein SH2-SH3 domains induce gene expression in a Ras-dependent fashion. Mol Cell Biol 1992; 12: 3425-30.

[5] Tocque B, Delumeau I, Parker F, Maurier F, Multon MC, Schweighoffer F. Ras-GTPase activating protein (GAP): a putative effector for Ras. Cell Signal 1997; 9: 153-8.

[6] Kennedy D, French J, Guitard E, Ru K, Tocque B, Mattick J. Characterization of G3BPs: tissue specific expression, chromosomal localisation and rasGAP binding studies. J Cell Biochem 2001; 84: 173-87.

[7] Guitard E, Parker F, Millon R, Abecassis J, Tocque B. G3BP is overexpressed in human tumors and promotes $S$ phase entry. Cancer Lett 2001; 162: 213-21.

[8] Zhang HZ, Liu JG, Wei YP, Wu C, Cao YK, Wang M Expression of G3BP and RhoC in esophageal squamous carcinoma and their effect on prognosis. World J Gastroenterol 2007; 13: 4126-30.

[9] Lapidot T, Dar A, Kollet O. How do stem cells find their way home? Blood 2005; 106: 1901-10.

[10] Wilson A, Trumpp A. Bone-marrow haematopoietic-stem-cell niches. Nat Rev Immunol 2006; 6: 93-106.

[11] Just I, Wilm M, Selzer J, et al. The enterotoxin from Clostridium difficile (ToxA) monoglucosylates the Rho proteins. J Biol Chem 1995; 270: 13932-6.

[12] Genth H, Hofmann F, Selzer J, Rex G, Aktories K, Just I. Difference in protein substrate specificity between hemorrhagic toxin and lethal toxin from Clostridium sordellii. Biochem Biophys Res Commun 1996; 229: 370-4.

[13] Hofmann WK, de Vos S, Elashoff D, et al. Relation between resistance of Philadelphia-chromosome-positive acute lymphoblastic leukaemia to the tyrosine kinase inhibitor STI571 and gene- expression profiles: a gene-expression study. Lancet 2002; 359: 481-6.

[14] Puccetti E, Zheng X, Brambilla D, et al. The integrity of the charged pocket in the $\mathrm{BTB} / \mathrm{POZ}$ domain is essential for the phenotype induced by the leukemia-associated $\mathrm{t}(11 ; 17)$ fusion protein PLZF/RARalpha. Cancer Res 2005; 65: 6080-8.

[15] Zheng X, Beissert T, Kukoc-Zivojnov N, et al. Gamma-catenin contributes to leukemogenesis induced by AML-associated translocation products by increasing the self-renewal of very primitive progenitor cells. Blood 2004; 103: 3535-43.

[16] Rüster B, Göttig S, Ludwig RJ, et al. Mesenchymal stem cells (MSCs) display coordinated rolling and adhesion behavior on endothelial cells. Blood 2006; 108: 3938-44.

[17] Bug G, Rossmanith T, Henschler R, et al. Rho family small GTPases control migration of hematopoietic progenitor cells into multicellular spheroids of bone marrow stroma cells. J Leukoc Biol 2002; 72: 837-45.

[18] Gottig S, Mobest D, Ruster B, et al. Role of the monomeric GTPase Rho in hematopoietic progenitor cell migration and transplantation. Eur J Immunol 2006; 36: 180-9.

[19] Lawrence MB, Springer TA. Leukocytes roll on a selectin at physiologic flow rates: distinction from and prerequisite for adhesion through integrins. Cell 1991; 65: 859-73.

[20] Peled A, Grabovsky V, Habler L, et al. The chemokine SDF-1 stimulates integrin-mediated arrest of CD34(+) cells on vascular endothelium under shear flow. J Clin Invest 1999; 104: 1199-211.

[21] Mohle R, Moore MA, Nachman RL, Rafii S. Transendothelial migration of $\mathrm{CD} 34+$ and mature hematopoietic cells: an in vitro study using a human bone marrow endothelial cell line. Blood 1997; 89: 72-80.

[22] Ghiaur G, Lee A, Bailey J, Cancelas JA, Zheng Y, Williams DA. Inhibition of RhoA GTPase activity enhances hematopoietic stem and progenitor cell proliferation and engraftment. Blood 2006; 108: 2087-94.

[23] Gu Y, Filippi MD, Cancelas JA, et al. Hematopoietic cell regulation by Rac1 and Rac2 guanosine triphosphatases. Science 2003; 302: 445-9.

[24] Cancelas JA, Lee AW, Prabhakar R, Stringer KF, Zheng Y, Williams DA. Rac GTPases differentially integrate signals regulating hematopoietic stem cell localization. Nat Med 2005; 11: 886-91.

[25] Price LS, Leng J, Schwartz MA, Bokoch GM. Activation of Rac and Cdc42 by integrins mediates cell spreading. Mol Biol Cell 1998; 9: 1863-71.

[26] Nobes CD, Hall A. Rho, rac, and cdc42 GTPases regulate the assembly of multimolecular focal complexes associated with actin stress fibers, lamellipodia, and filopodia. Cell 1995; 81: 53-62.

[27] Pazman C, Mayes CA, Fanto M, Haynes SR, Mlodzik M. Rasputin, the Drosophila homologue of the RasGAP SH3 binding protein, functions in ras- and Rho-mediated signaling. Development 2000; 127: $1715-25$.

This is an open access article licensed under the terms of the Creative Commons Attribution Non-Commercial License (http://creativecommons.org/licenses/ by-nc/3.0/) which permits unrestricted, non-commercial use, distribution and reproduction in any medium, provided the work is properly cited. 